\title{
THE EFFECT OF THIN CARBON AND CHEMOMECHANICALLY SOFTENED LAYERS ON THE NEAR SURFACE MECHANICAL PROPERTIES OF SAPPHIRE TESTED BY NANOINDENTATION
}

\author{
S J Bull and A Yadav, School of Engineering, Newcastle University, Newcastle upon Tyne, \\ NE17RU, UK.
}

Keywords: Chemomechanical effects; Implantation; Mechanical properties; Sapphire

\begin{abstract}
The mechanical properties of the near-surface region of oxide ceramics such as sapphire are affected by adsorbate-induced changes to dislocation mobility that can be assessed by nanoindentation with contact depth less than 30nm. Modification of this chemomechanical behaviour of the surface of sapphire by titanium ion implantation has been investigated in this study. At high doses, a soft surface amorphous layer has formed due to implantation-induced damage. However, hardness increases at low implanted doses, which is associated with implantation-induced damage but that also chemomechanical softening of the surface is reduced due to the removal of adsorbed water. For the optimum implanted dose the water readsorption does not recur even several years after the implantation treatment. This is associated with the formation of a thin, hydrophobic carbonaceous layer on the surface. This is important when designing ceramic devices such as orthopaedic implants and fibre guides that operate in an aqueous environment.
\end{abstract}

\section{Introduction}

Chemomechanical effects due to long-term environmental exposure are known to exert a considerable influence on the mechanical properties of ceramic surfaces [1-5]. Over past few decades studies have consistently reported that the mechanical properties such as creep, fracture and fatigue have significant changes due to the test environment [6]. In particular, more recent studies have shown that the chemomechanical effect has significant influence on hardness in smallscale tests on various ceramic surfaces measured by high-resolution techniques such as Nanoindentation [7-10]. This chemomechanical effect can be prevented by modifying the surface layer by ion implantation which is also expected to improve the surface mechanical properties under certain treatment conditions $[11,12]$. Surface modification of ceramic surfaces by ion implantation has been investigated to tailor the mechanical properties of the thin surface layers for tribological applications [11-13]. Ion implantation of ceramic materials can lead to either nearsurface hardening or softening [14-15]. The hardening is attributed to combination of radiationhardening and solid solution effects as above whilst the softening is usually a consequence of amorphization.

In this work, we systematically investigated how the chemomechanical behaviour of sapphire changes after $\mathrm{Ti}^{+}$ion implantation. The nanoindentation hardness is highly dependent on the implanted dose and the environment is only important in a critical dose range where removal of adsorbed water layer has occurred. Thus treating the surface with appropriate ion implanted species can control the near-surface hardness and reduce the chemomechanical effect which is required for optimum tribological performance. 


\section{Experimental}

The single crystal sapphire (10 $\overline{1} 2)$ substrates were obtained from GEC and cleaved into smaller sizes of $10 \times 10 \times 0.5 \mathrm{~mm}$. Prior to implantation, the specimens were carefully ultrasonically cleaned degreased in isopropyl alcohol. The specimens were implanted with titanium $(300 \mathrm{keV})$ to doses in the range from $2 \times 10^{16}-1.5 \times 10^{17} \mathrm{Ti}^{+} \mathrm{cm}^{-2}$ in the Cockcroft-Walton facility at UKAEA Harwell. All implantations were carried out at room temperature with a beam current of few microamps per centimetre squared; beam heating was estimated to be about $250{ }^{\circ} \mathrm{C}$. Under these conditions the ion range is $159 \mathrm{~nm}$ and its straggle is $43 \mathrm{~nm}$ according to calculations using the SRIM. The depth of maximum damage is at $126 \mathrm{~nm}$ and the sputtering yield is 2.6.

The ion depth profiles were evaluated using 2.0 MeV $\alpha$-particle Rutherford Backscattering (RBS) spectra measurements with the sample normal oriented at about $7^{\circ}$ to the analysis beam direction. This generates the spectrum from a "randomly oriented" sample. Channelled RBS was then used to determine the profile of damage caused by the implantation. In this case the [0001] channel at about $58^{\circ}$ to the surface normal was aligned with the beam direction. In this orientation the open channels in the crystallographic structure are aligned with the analysis beam and penetration of the beam is increased and backscattering is reduced. Only when damage disrupts the channel will the backscattered signal increase.

To characterise the near surface mechanical properties of the sapphire with different doses of implantation, nanoindentation experiments were performed in the displacement controlled mode using a Hysitron Triboindenter nanoindentation system fitted with a sharp Berkovich indenter (tip end radius $100 \mathrm{~nm}$ ). To investigate the environment effects that influence the hardness, the nanoindentation tests were performed on the same sapphire sample after soaking the surface in methanol or water for $24 \mathrm{hrs}$. After the samples were removed from the liquids, they were dried immediately at $50{ }^{\circ} \mathrm{C}$ using a hot air blower. Indentation tests were performed immediately after drying. An array of twenty indents was created on every sample.

The chemical compositions and valence states of sapphire and sapphire implanted with $\mathrm{Ti}$ were studied by x-ray photoelectron spectroscopy measurements (XPS). All the spectra were acquired with a Kratos Axis Nova spectrometer. The samples were mounted on a clean Al platen and immobilised with carbon tape and copper clips. The largest analysis area available in the instrument $(300 \times 700 \mu \mathrm{m})$ was used in all the measurements. All measurements were replaced on three different analysis positions with non-overlapping analysis areas on each sample. Charge compensation was used throughout the measurement. The XPS spectra were analysed using the Casa XPS software.

Surface polarity and changes to surface energy after implantation were investigated using water drop contact angles measured using an optical tensiometer. Prior to testing surfaces were cleaned with isopropyl alcohol and a $10 \mu \mathrm{L}$ drop of deionised water was used for measurement in each case. Five drops were measured on different areas of each surface tested and the results were averaged.

\section{Results and Discussion}

\section{Characterisation of the implant profiles and damage}

Figure 1a presents the random and (1012) channelled RBS spectra from the unimplanted sapphire surface. It can be observed that the random spectrum does not coincide with channelled spectrum. The crystal quality of the starting material can be determined from the ratio of the channelled 
scattering yield to the random scattering yield just below the two surface peaks. In this case, for $\mathrm{Al}$ the aligned yield is $\sim 50 \%$ of the random yield and for $\mathrm{O} \sim 55 \%$, which indicate that the single crystalline sapphire surface is almost damage free as might be expected for semiconductor-grade material. The effect of implanting $\mathrm{Ti}^{+}$(to $3.2 \times 10^{16} \mathrm{Ti}^{+} \mathrm{cm}^{-2}$ ) into the sapphire is shown in Fig. $1 \mathrm{~b}$. Comparing the (0001) channelled yields from the implanted and unimplanted regions, a substantial disorder has been introduced in both the $\mathrm{Al}$ and $\mathrm{O}$ subarrays but the random and channelled spectra do not coincide. This indicates that the near-surface region has not become amorphous.
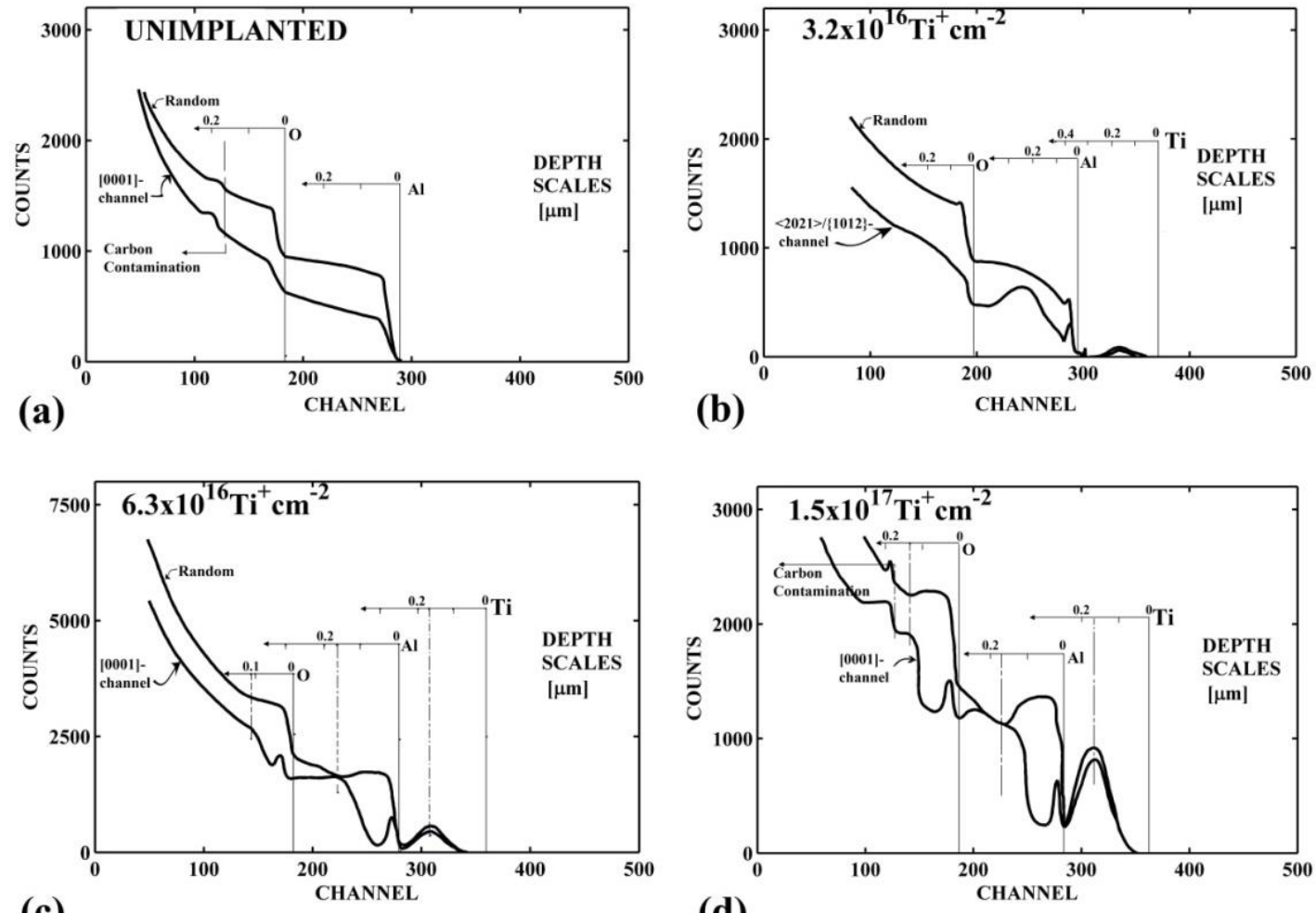

(c)

(d)

Figure 1. A sequence of channelled and random RBS spectra obtained from sapphire (a) unimplanted, (b) implanted with $3.2 \times 10^{16} \mathrm{Ti}^{+} \mathrm{cm}^{-2}$, (c) implanted with $6.3 \times 10^{16} \mathrm{Ti}^{+} \mathrm{cm}^{-2}$, and (d) implanted with $1.5 \times 10^{17} \mathrm{Ti}^{+} \mathrm{cm}^{-2}$.

Figure 1c shows the random and channelled spectra for the implantation dose of $6.3 \times 10^{16}$ $\mathrm{Ti}^{+} \mathrm{cm}^{-2}$ in sapphire. The peak in the titanium concentration profile occurs at a greater depth which is evidence that amorphisation has occurred (ions travel a greater distance in amorphous material with lower density). At this dose, the surface peaks from $\mathrm{Al}$ and $\mathrm{O}$ are considerably larger than in the case of previous doses. The near-surface region (just behind the surface peak) is damaged and shows complete amorphisation. The depth at which the damage saturates in the Al subarray corresponds reasonably well with the peak of the Ti distribution. The implanted Ti shows a small channelling effect (the channelled yield is $\sim 88 \%$ of the random yield). An RBS spectrum from sapphire implanted with $\mathrm{Ti}^{+}$ions to a dose of $1.5 \times 10^{17} \mathrm{Ti}^{+} \mathrm{cm}^{-2}$ is shown in Fig. 1d. It is observed that both the random and channelled spectra coincide with each other and significant disorder in both the $\mathrm{Al}$ and $\mathrm{O}$ subarrays is observed. The near surface region is completely damaged. nevertheless, the Ti peak is found to be still Gaussian. Thus with increasing dose more and more damage is produced. 
To summarize, at the low doses both the random and channelled spectra do not coincide whereas for the higher doses they coincide which implies that an amorphous layer has been formed at high doses. None of the specimens had been rendered amorphous at low doses, this being in agreement with earlier work [16]. The damaged regions become more predominant as the dose increases. For all the implanted species, we have not observed a completely disordered surface region up to doses of $6.3 \times 10^{16} \mathrm{Ti}^{+} \mathrm{cm}^{-2}$, whereas doses of $1.5 \times 10^{17} \mathrm{Ti}^{+} \mathrm{cm}^{-2}$ would be sufficient to turn the near-surface region completely amorphous. In all cases a little damage is visible in the $\mathrm{Al}$ and $\mathrm{O}$ subarrays and the Ti implanted peak remains approximately Gaussian. As the implantation doses are increased the implanted profile moves away from the surface which is due to the transformation from crystalline to amorphous surface with lower density [16].

\section{Nanoindentation measurements}

In our previous studies [9] we have shown that the near surface hardness of sapphire for both micro- and nanoindentation is reduced after exposure to water and this reduction in hardness was consistent in both techniques. In contrast, the hardness is found to increase after exposure to methanol or ethanol as the contact depth is reduced. This is the result of the normal "indentation size effect" which is usually found when testing across different length scales in ceramics [1718]. However, the reduction in surface hardness after exposure to the water was directly associated with a soft water adsorbate-affected surface layer [9]. The indentation size effect hardening can be completely removed or even reversed due to this chemomechanical effect.

For the implanted samples the hardness at the high dose $\left(2.5 \times 10^{16} \mathrm{Ti}^{+} \mathrm{cm}^{-2}\right)$ is higher than that from samples implanted with low dose $\left(1 \times 10^{16} \mathrm{Ti}^{+} \mathrm{cm}^{-2}\right)$. Furthermore, it is observed that below the contact depth $40 \mathrm{~nm}$, there is a significant variation in hardness after exposure to water whereas this is not seen in the case of the $2.5 \times 10^{16} \mathrm{Ti}^{+} \mathrm{cm}^{-2}$ dose (Figures. 2a and 2b). This suggests that the adsorbed water is removed at a dose of $2.5 \times 10^{16} \mathrm{Ti}^{+} \mathrm{cm}^{-2}$ and adsorbate-induced increases in dislocation mobility are hence reduced. The effect of removal of any chemomechanical softening at low doses is much greater than any solid solution or radiation damage hardening from the implantation process.
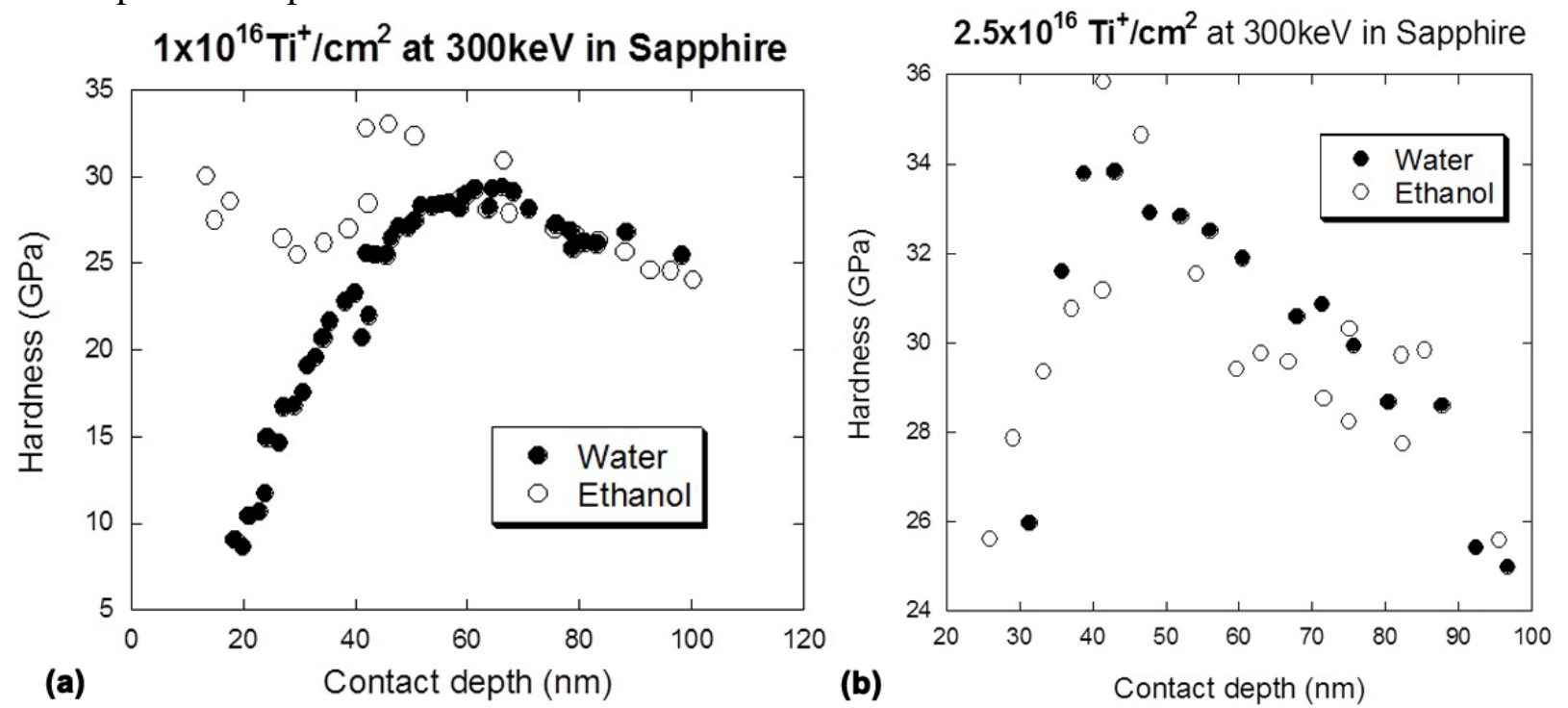

Figure 2. Comparison in hardness vs contact depth after ethanol and water exposure for sapphire samples implanted with (a) $1 \times 10^{16} \mathrm{Ti}^{+} \mathrm{cm}^{-2}$ and (b) $2.5 \times 10^{16} \mathrm{Ti}^{+} \mathrm{cm}^{-2}$. 
300keV Ti Implantation in Sapphire

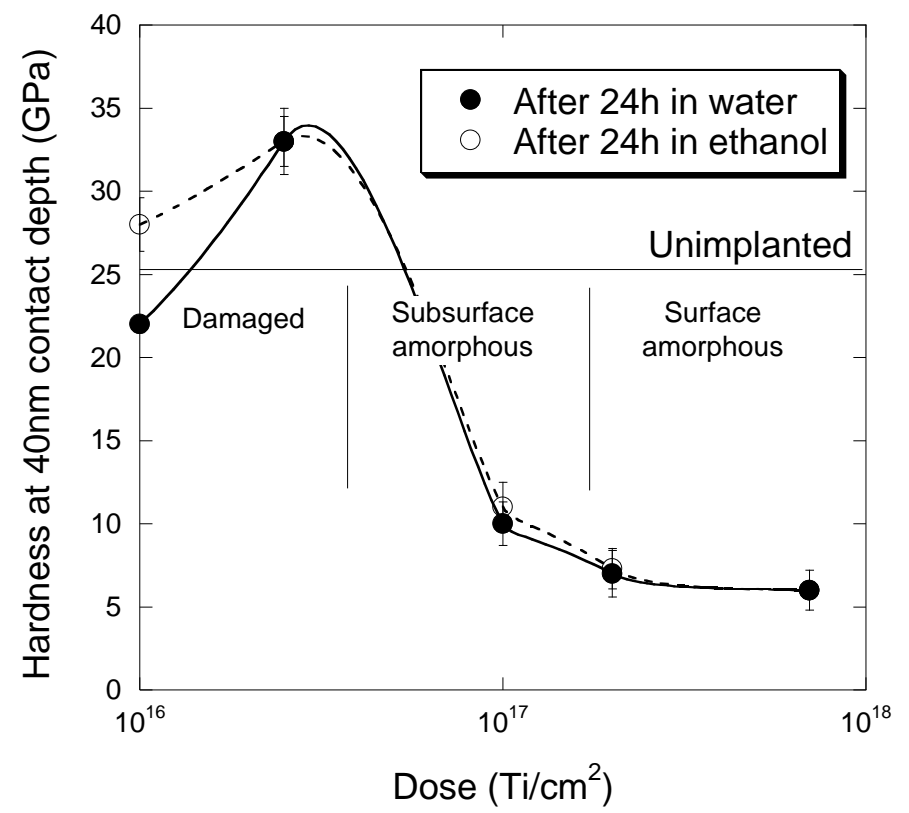

Figure 3. Hardness at the contact depth $40 \mathrm{~nm}$ with respect to dose of various $\mathrm{Ti}^{+}$ion implantations in sapphire after water and ethanol exposure.

As we have seen from the above (Figure 2a) the hardness changes only below the contact depth of $40 \mathrm{~nm}$ due to chemomechanical effects. Therefore, we have compared the hardness at this threshold contact depth $(40 \mathrm{~nm})$ for samples with different $\mathrm{Ti}^{+}$ion implantation doses (Figure 3 ) after ethanol and water exposure. The hardness at the low doses $\left(<3 \times 10^{16} \mathrm{Ti}^{+} \mathrm{cm}^{-2}\right)$ is higher and starts increasing as the implantation dose increases up to a maximum which is associated with the onset of amorphisation (subsurface amorphous). It decreases further as the implantation dose reaches $10^{17} \mathrm{Ti}^{+} \mathrm{cm}^{-2}$ and eventually it becomes almost constant which is the regime of complete surface amorphisation. In water, after low dose $\mathrm{Ti}^{+}$ion implantation the hardness of implanted sapphire is increased in comparison to unimplanted sapphire. This is to be expected mainly due to the reduction in the chemomechanical softening effect after the implantation although a relatively small radiation damage hardening contribution is also observed.

\section{Changes to the sapphire surface due to ion implantation}

It has been suggested that the chemomechanical effect in sapphire is related to the presence of absorbates which affect dislocation mobility [9] and therefore it might be expected that these adsorbates are sputtered away by the implantation process. However, the fact that the changes to chemomechanical behaviour persist even after a long exposure to the atmosphere after implantation suggests that a permanent change to the sapphire surface has occurred which is not seen in unimplanted material. Thus it is important to understand the changing surface chemistry as well as the structural changes already identified.

Furthermore, there is a change in surface energy after implantation which also persists. The water drop contact angles were measured for unimplanted sapphire and the titanium implanted sapphire at three two different implantation doses. For unimplanted sapphire the contact angle was $67 \pm 3^{\circ}$ which is in the expected range for sapphire surfaces cleaned in water or alcohol. After implantation to $2.5 \times 10^{16} \mathrm{Ti}^{+} \mathrm{cm}^{-2}$, where the sapphire near surface layer is damaged but crystalline 
the contact angle increases to $95 \pm 5^{\circ}$ and the surface is clearly more hydrophobic. By the time that the amorphous layer has reached the surface, at a dose of $1.5 \times 10^{17} \mathrm{Ti}^{+} \mathrm{cm}^{-2}$ the contact angle has fallen to $79 \pm 3^{\circ}$ indicating an increase in hydrophilicity compared to the lose dose implantation but not to the same extent as the unimplanted sapphire.

In order to understand the change in contact angle, XPS was carried out to characterise the surface chemical composition (Fig. 4). The C 1S and Ti 2P spectrum were fitted using Gaussian functions superimposed on a Shirley and linear background respectively. For the Ti implanted samples, the $\mathrm{C} 1 \mathrm{~S}$ peaks observed at the binding energy of $284.80 \mathrm{eV}$ and $286.92 \mathrm{eV}$ corresponding to $\mathrm{C}-\mathrm{C}$ and $\mathrm{C}-\mathrm{O}$ bonds are shown in Figs. $4 \mathrm{a}$ and $4 \mathrm{c}$, whereas for unimplanted sapphire the $\mathrm{C} 1 \mathrm{~S}$ peak at $285.41 \mathrm{eV}$ and $287.99 \mathrm{eV}$ can be assigned to $\mathrm{C}-\mathrm{C}$ and $\mathrm{C}=\mathrm{O}$ respectively (Fig. 4e). The increased peak areas for the implanted samples show that there is considerably more carbon on the surface. The Ti $2 \mathrm{P}$ spectra of the $1 \times 10^{17} \mathrm{Ti}^{+} \mathrm{cm}^{-2}$ showed the $\mathrm{Ti} 2 \mathrm{P} 3 / 2$ and $\mathrm{Ti} 2 \mathrm{P} 1 / 2$ intensity maxima at $458.86 \mathrm{eV}$ and $464.54 \mathrm{eV}$, respectively (Fig. 4b), whereas the Ti $2 \mathrm{P}$ spectra of the $3.2 \times 10^{16} \mathrm{Ti}^{+} \mathrm{cm}^{-2}$ has low intensity as the surface concentration is too small to observe (Fig. $4 \mathrm{~d}$ ). All the data indicate that it is the carbon at the surface which controls the surface adsorbate and chemomechanical behaviour of the sapphire after titanium implantation. The question then arises about the origin of this carbon which was found to be present immediately after implantation of fresh samples and therefore is not likely to be environmental contamination.
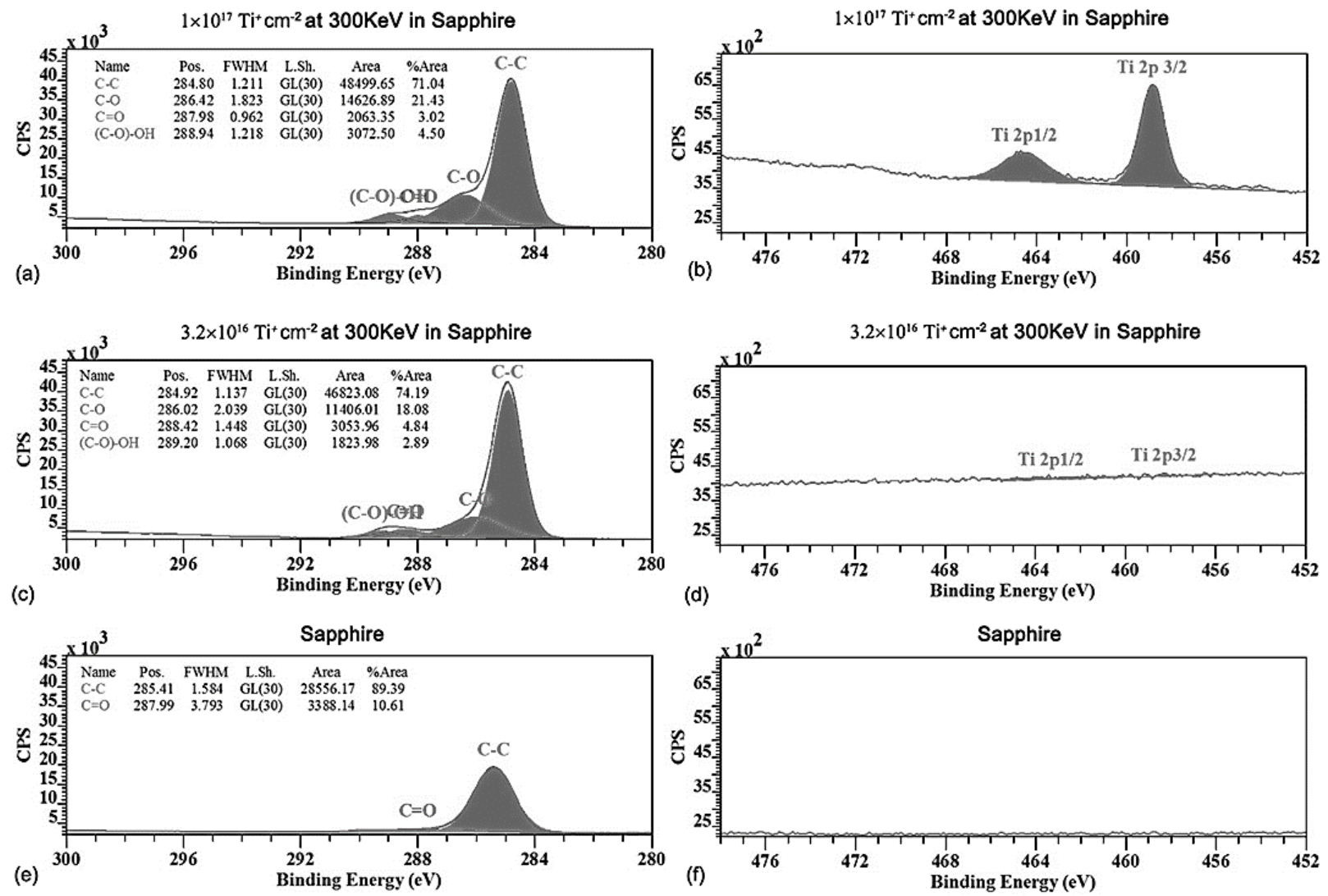

Figure 4. (a) C 1S and (b) Ti 2P XPS spectra of sapphire implanted with $1 \times 10^{17} \mathrm{Ti}^{+} \mathrm{cm}^{-2}$, (c) C $1 \mathrm{~S}$ and (d) Ti 2P XPS spectra of sapphire implanted with $3.2 \times 10^{16} \mathrm{Ti}^{+} \mathrm{cm}^{-2}$, and (e) C $1 \mathrm{~S}$ and (f) $\mathrm{Ti}$ 2P XPS spectra of sapphire. 
The origin of the carbon is due to the interaction of the ion beam with carbon species depositing on the sapphire surface in the vacuum chamber. The sample chamber used was pumped by a diffusion pump to a base pressure of $10^{-6}$ Torr. Back-streaming of diffusion pump oil (Santovac5 - a five ring polyphenyl ether) leads to the deposition of carbon and oxygen containing species on the surface of the samples with an estimated layer thickness of a few nanometres. The ion beam deposits energy in inelastic collisions in the near-surface region resulting in bond breakage and structural rearrangement in the adsorbed layers. TRIM calculations for a $5 \mathrm{~nm}$ layer of diffusion pump oil on a sapphire surface irradiated with a $300 \mathrm{keV}$ titanium ion beam show considerable recoil damage in the oil layer (mainly carbon and hydrogen) and preferential sputtering of hydrogen. The highly reactive damaged carbon the forms new bonds to carbon in the surface layer and to oxygen from the sapphire and contains a small amount of implanted titanium. The result is a highly stable, non-polar, carbonaceous surface layer on top of the sapphire. The stability of the surface layer is evidenced by the fact that no change in properties or composition was observed even several years after the implantation.

The presence of a non-polar carbon layer on the sapphire surface will thus reduce adsorbate concentration and result in high hardness. High surface hardness is observed more for low doses (Figure 3) because the concentration of defects is not high enough to cause amorphisation but a non-polar surface is produced. In case of higher doses (above $\sim 3 \times 10^{16} \mathrm{Ti}^{+} \mathrm{cm}^{-2}$ ) amorphisation occurs. The surface hardness is less above this doses because amorphous material is softer than crystalline material but does not show chemomechanical effects because such amorphous material does not support dislocation-based deformation mechanisms.

\section{Conclusions}

The removal of environmentally softened layers on the sapphire surface after low dose $\mathrm{Ti}^{+}$ion implantation has been demonstrated. It has been shown to result in improving near-surface hardness by reducing chemomechanical effects. The low load hardness of sapphire is influenced by the dose of $\mathrm{Ti}^{+}$ion implantation in a complex manner. Implantation is found to increase the hardness up to the onset of amorphisation $\left(\sim 3 \times 10^{16} \mathrm{Ti}^{+} \mathrm{cm}^{-2}\right)$ whilst at higher doses hardness is reduced. The increase in near-surface hardness is partly due to the removal of physisorbed water which promotes chemomechanical softening of the sapphire substrate. The removal of the adsorbed water layer is due to the formation of a non-polar, carbonaceous layer on the sapphire surface by ion beam cracking of adsorbed diffusion pup oil. Based on this study, it is concluded that ion implantation with an appropriate ion species can control the chemomechanical effects and therefore can control the near-surface mechanical properties of sapphire.

\section{Acknowledgements}

This work was supported by EPSRC through the North East Centre for Energy Materials grant.

\section{References}

1. A.R.C. Westwood, R.D. Huntington, and N.H Macmillan, Influence of environment on the mobility of near-surface dislocations in ionic crystals, J. Appl. Phys., 44 (1973) 5194-5195.

2. J.T. Czernuszka and T.F. Page, The importance of microscopy in studying the wear behaviour of ceramic surfaces, J. Microsc., 140 (1985) 159-169. 
3. A.R.C. Westwood, N.H. Macmillan and R.S. Kalyoncu, Environment-Sensitive Hardness and Machinability of $\mathrm{Al}_{2} \mathrm{O}_{3}$, J. Am. Ceram. Soc., 56 (1973) 258-262.

4. N.H. Macmillan, R.D. Huntington, and A.R.C. Westwood, Chemomechanical control of sliding friction behaviour in non-metals, J. Mater. Sci., 9 (1974) 697-706.

5. A.R.C. Westwood, J.S. Ahearn, and J.J. Mills, Developments in the theory and application of chemomechanical effects, Colloids Surf., 2 (1981)1-35.

6. R.E. Hanneman and J.H. Westbrook. Effects of adsorption on the indentation deformation of non-metallic solids, Philos. Mag., 1 8(1968) 73-88.

7. J.T. Czernuszka and T.F. Page, Characterizing the surface contact behaviour of ceramics, J. Mater. Sci., 22 (1987) 3907-3916.

8. Hainsworth SV, Page TF (1994) Nanoindentation studies of the chemomechanical effect in sapphire, J Mater Sci 29:5529-5540.

9. S.J. Bull et al The origins of chemomechanical effects in the low-load indentation hardness and tribology of ceramic materials, J. Mater. Sci., 51 (2016) 107-125.

10. S.V. Hainsworth and T.F. Page, Nanoindentation studies of chemomechanical effects in thin film coated systems, Surf. Coat. Technol., 68 (1994) 571-575.

11. P.J. Burnett and T.F. Page, Chemomechanical effect in ion-implanted magnesium oxide, J. Mater. Sci. Lett., 4 (1985) 1364-1370.

12. S.J. Bull and T.F. Page, Chemomechanical effects in ion-implanted MgO, J. Phys. D Appl. Phys. 22 (1989) 941-944.

13. N.E.W. Hartley, Ion implantation and surface modification in tribology, Wear, 34 (1975) $427-438$.

14. P.J. Burnett and T.F. Page, Surface softening in silicon by ion implantation, J. Mater. Sci., 19 (1984) 845-860.

15. H. Naramoto et al, Ion implantation and thermal annealing of $\alpha-\mathrm{Al}_{2} \mathrm{O}_{3}$ single crystals, J. Appl. Phys., 54 (1983) 683-698.

16. P.J. Burnett and T.F. Page, The friction and hardness of ion-implanted sapphire, Wear 114 (1987) 85-96.

17. S.J. Bull, T.F. Page and E.H. Yoffe, An explanation of the indentation size effect in ceramics, Philos. Mag. Lett., 59 (1989) 281-288.

18. S.J. Bull, On the origins and mechanisms of the indentation size effect Z. Für. Met., 94 (2003) 787-792. 